Pacific Journal of Mathematics

QUALITATIVE BEHAVIOR OF SOLUTIONS OF ELLIPTIC
FREE BOUNDARY PROBLEMS 


\title{
QUALITATIVE BEHAVIOR OF SOLUTIONS OF ELLIPTIC FREE BOUNDARY PROBLEMS
}

\author{
KIRK E. LANCASTER
}

A general free boundary problem is investigated and the qualitative behavior of the fixed boundary is compared with that of the fixed boundary. As an illustration, consider the following situation. Let $\Gamma^{*}$ be a given Jordan curve in $\mathfrak{R}^{2}$. For each Jordan curve $\Gamma$ in $\mathfrak{R}^{2}$ which surrounds $\Gamma^{*}$, we let $\Omega=\Omega\left(\Gamma^{*}, \Gamma\right)$ be the region between $\Gamma^{*}$ and $\Gamma$. Let $Q$ be the second-order elliptic operator given by

$$
Q u \equiv a u_{x x}+2 b u_{x y}+c u_{y y} \quad \text { in } \Omega
$$

where $a, b, c$ depend on $x, y, u_{x}$, and $u_{y}$ and $a c-b^{2}>0$. Consider the free boundary problem of finding a curve $\Gamma$ and a function $u \in C^{2}(\Omega) \cap C^{1}(\Omega \cup \Gamma) \cap C^{0}(\bar{\Omega})$ such that

$$
\begin{aligned}
Q u=0 & \text { in } \Omega \\
u=1 & \text { on } \Gamma^{*}
\end{aligned}
$$

and, for a fixed $\lambda>0$,

$$
u=0, \quad|\nabla u|=\lambda \quad \text { on } \Gamma,
$$

where $\Omega=\Omega\left(\Gamma^{*}, \Gamma\right)$. Suppose $\Gamma$ and $u$ constitute a solution of this free boundary problem. Using curves of constant gradient direction, the geometry of the free boundary $\Gamma$ is compared to the geometry of the fixed boundary $\Gamma^{*}$. In particular, $\Gamma$ is shown to have a "simpler" geometry than does $\Gamma^{*}$.

0. Introduction. Let $a, b, c \in C^{0}\left(\mathfrak{R}^{4}\right)$ with $a c-b^{2}>0$ in $\mathfrak{R}^{4}$ and define $Q$ to be the quasilinear, elliptic, second-order partial differential operator given by

$$
Q u=a u_{x x}+2 b u_{x y}+c u_{y y}
$$

for $u \in C^{2}$, where $a=a(x, y, p, q), b=b(x, y, p, q), c=$ $c(x, y, p, q)$ and $p=u_{x}(x, y), q=u_{y}(x, y)$. We are interested in the following free boundary problem.

Quasilinear free boundary problem. Given $\Gamma^{*}$ a Jordan curve in $\mathfrak{R}^{2}$ or a finite collection of pairwise disjoint Jordan curves in $\mathfrak{R}^{2}$ and a number $\lambda>0$, find a bounded domain $\Omega \subset \mathfrak{R}^{2}$, a finite collection $\Gamma$ 
of pairwise disjoint Jordan curves in $\mathfrak{R}^{2}$, and a function $u \in C^{2}(\Omega) \cap$ $C^{1}(\Omega \cup \Gamma) \cap C^{0}(\bar{\Omega})$ such that $\Gamma \cap \Gamma^{*}=\varnothing, \partial \Omega=\Gamma \cup \Gamma^{*}$, and

$$
\begin{array}{ll}
Q u=0 & \text { in } \Omega, \\
u=1 & \text { on } \Gamma^{*}, \\
u=0 & \text { on } \Gamma, \\
|\nabla u|=\lambda & \text { on } \Gamma .
\end{array}
$$

We are also interested in the following related free boundary problem. Let $F \in C^{0}\left(\mathfrak{R}^{2} \times \mathfrak{R}^{2} \times \mathfrak{R}^{2 \times 2}\right)$ satisfy:

$F(x, y, P, R)$ is locally uniformly Lipschitz with respect to the $P \in \mathfrak{R}^{2}$ and $R \in \mathfrak{R}^{2 \times 2}$ variables;

$F$ is elliptic;

$\left|F_{P}\right|$ is locally bounded;

$F(x, y, P, 0)=0$ for all $(x, y) \in \mathfrak{R}^{2}$ and $P=(p, q) \in \mathfrak{R}^{2}$, where $\mathfrak{R}^{2 \times 2}$ denotes the 3 -dimensional space of real, symmetric $2 \times 2$ matrices (see [12], pp. 441-446). Let $\mathfrak{I}$ be the elliptic, fully nonlinear partial differential operator of second-order depending on $x, y, D u$, $D^{2} u$ given by

$$
\mathfrak{I} u=F(x, y, P, R)
$$

with $P=D u$ and $R=D^{2} u \in \mathfrak{R}^{2 \times 2}$.

Fully nonlinear free boundary problem. Given $\Gamma^{*}$ a Jordan curve in $\mathfrak{R}^{2}$ or a finite collection of pairwise disjoint Jordan curves in $\mathfrak{R}^{2}$ and a number $\lambda>0$, find a bounded domain $\Omega \subset \mathfrak{R}^{2}$, a finite collection $\Gamma$ of pairwise disjoint Jordan curves in $\Re^{2}$, and a function $u \in C^{2}(\Omega) \cap$ $C^{1}(\Omega \cup \Gamma) \cup C^{0}(\bar{\Omega})$ such that $\Gamma \cap \Gamma^{*}=\varnothing, \partial \Omega=\Gamma \cup \Gamma^{*}$, and

$$
\begin{array}{ll}
\mathfrak{I} u=0 & \text { in } \Omega, \\
u=1 & \text { on } \Gamma^{*}, \\
u=0 & \text { on } \Gamma, \\
|\nabla u|=\lambda & \text { on } \Gamma .
\end{array}
$$

We will call $\Gamma^{*}$ the fixed boundary and $\Gamma$ the free boundary of this problem. We should note that the requirement that $\Omega$ be bounded can be relaxed.

A number of authors have considered questions about certain qualitative aspects of the free boundary. For example, D. Tepper ([20], [21]; see also [8], pp. 432-443) proved that if $Q=\Delta, \Omega$ is an annular domain, and $\Gamma^{*}$ is convex or starlike, then $\Gamma$ is also convex or star- 
like. For an inhomogeneous free boundary problem, T. Vogel ([22]) obtained the convexity or starlikeness of $\Gamma$ when $\Gamma^{*}$ is convex or starlike. For parabolic free boundary problems, K. Nickel ([14], [15]) considered profiles of solutions of the heat equation and A. Friedman and R. Jensen ([10]) obtained the convexity of the free boundary in Stefen and dam problems.

In 1983 (approximately), A. Acker ([1]) and A. Friedman-T. Vogel ([11]) independently considered the case $Q=\Delta$ (i.e. $u$ is harmonic in $\Omega$ ) and used curves of constant gradient direction to obtain qualitative information about the geometry of the free boundary in terms of the geometry of the fixed boundary when $\Omega$ is (equivalent to) a doubly connected or annular domain (i.e. $\Gamma$ and $\Gamma^{*}$ are Jordan curves with one of the curves lying inside the other); these curves of constant gradient direction are related to "nodal lines" ([9]; see also [10], [14], [17]). Vogel ([22]) also used these curves to examine a galvanization problem. Subsequently, Acker showed that curves of constant gradient direction can be a powerful tool for investigating the geometry of free boundaries by obtaining more detailed qualitative information about the free boundary in terms of information about the fixed boundary ([2], [3]) and obtaining qualitative results without the assumption that $\Omega$ is doubly connected ([4]). Further, Acker, together with the author, used this method to study free boundary problems for parametric minimal surfaces in $\mathfrak{R}^{3}$ ([6]) and the one-dimensional heat equation ([7]). Acker ([5]) has also found examples in $\mathfrak{R}^{n}, n>2$, which show that the qualitative results above are generally false in three or more dimensions.

We will show that the "method of curves of constant direction" can be used to investigate the quasilinear and fully nonlinear free boundary problems for any operator $Q$ or $\mathfrak{I}$ as given by $(1)$ or $\left(1^{\prime}\right)$ respectively. We will prove that each component of $\Gamma$ has a "simpler" geometry than does $\Gamma^{*}$. For example, when $\Omega$ is an annular domain, we will prove that the total curvature, the number of local maxima (minima) with respect to a prescribed direction $\vec{\nu}$, and the number of inflection points of $\Gamma$ are less than or equal to the total curvature, the number of local maxima (minima) with respect to $\vec{\nu}$, and the number of inflection points of $\Gamma^{*}$ respectively. When we do not assume $\Omega$ has a particular topological structure, we see that total "positive curvature" and the number of $\vec{\nu}$-minima of $\Gamma$ are less than or equal to the total "positive curvature" and the number of $\vec{\nu}$-minima of $\Gamma^{*}$ respectively. We note that all of the qualitative results of Acker for 
the harmonic free boundary problem obtained using curves of constant gradient direction remain valid for our free boundary problems. Since, in addition to the free boundary problems $(2)$ and $\left(2^{\prime}\right)$, this method has proven useful for inhomogeneous ([22], [3]), parabolic ([7]), and axial-symmetric ([5]) free boundary problems, we suspect that curves of constant gradient direction will eventually prove useful for investigating a number of additional elliptic and parabolic free boundary problems.

1. Main results. We will adopt the notation of [2] and [4]. Suppose $\Gamma^{*}$ and $\lambda$ are given and $\Omega, \Gamma, u$ constitute a solution of the free boundary problem (2). We assume $\Gamma^{*}$ is a $C^{1}$ curve or union of curves and has bounded curvature. We will orient $\partial \Omega$ so that the forward direction on $\partial \Omega$ is such that $\Omega$ lies locally to the left of $\Omega$ and locally to the right of $\Gamma^{*}$ (e.g. Figure 1, [4]). We let $\vec{n}(x, y)$ denote the unit normal vector to $\partial \Omega$ at $(x, y) \in \partial \Omega$ which points to the left; hence $\nabla u(x, y)=|\nabla u(x, y)| \vec{n}(x, y)$ for $(x, y) \in \partial \Omega$. We will assume that for each unit vector $\vec{e}$, the curve $\Gamma^{*}$ contains at most finitely many maximal segments (including isolated points) on which $\vec{n}(x, y)=\vec{e}$.

Definition. Given a unit vector $\vec{\nu}$, we call $\left(x_{0}, y_{0}\right) \in \Gamma$ a $\vec{\nu}$ minimum ( $\vec{\nu}$-maximum) of $\Gamma$ if $\vec{n}\left(x_{0}, y_{0}\right)=\vec{\nu}$ and $\left(x_{0}, y_{0}\right)$ is a strict local minimum (maximum) relative to $\Gamma$ of $f(x, y)=\vec{\nu} \cdot(x, y)$ (see, for example, Figures 2 and 3, [4]).

Definition. Given a unit vector $\vec{\nu}$, we call $\left(x_{0}, y_{0}\right) \in \Gamma^{*}$ a $\vec{\nu}$ minimum ( $\vec{\nu}$-maximum) of $\Gamma^{*}$ if $\vec{n}\left(x_{0}, y_{0}\right)=\vec{\nu}$ and either $\left(x_{0}, y_{0}\right)$ is a strict local minimum (maximum) relative to $\Gamma$ of $f(x, y)$ $=\vec{\nu} \cdot(x, y)$ or there is a closed line segment $\gamma^{*} \subset \Gamma^{*}$ such that $\left(x_{0}, y_{0}\right) \in \gamma^{*}$ and $\vec{\nu} \cdot(x, y)>(<) \vec{\nu} \cdot\left(x_{0}, y_{0}\right)$ for $(x, y) \in \Gamma^{*} \backslash \gamma^{*}$ near $\gamma^{*}$. Here $\gamma^{*}$ is considered as a single local extremum.

Definition. Given $\sigma \subset \Gamma$, we say that $\gamma$ has positive (negative) curvature on $\sigma$ if and only if $\Gamma$ has nonvanishing curvature on $\sigma$ and for each $(x, y) \in \sigma$, there exists $r>0$ such that the set of points which are within $r$ of $(x, y)$ and lie to the left (right) of $\partial \Omega$ is a convex set. We define the notation of positive and negative curvature of $\Gamma^{*}$ similarly.

Definition. We call $(x, y) \in \partial \Omega$ a positive (negative) inflection point of $\partial \Omega$ if and only if $\partial \Omega$ has negative (positive) curvature locally before $(x, y)$ and has positive (negative) curvature locally after 
$(x, y)$ or there is a line segment $\sigma \subset \Gamma^{*}$ with $(x, y) \in \sigma$ such that $\Gamma^{*}$ has negative (positive) curvature locally before $\sigma$ and positive (negative) curvature locally after $\sigma$.

THEOREM 1. Let $\Gamma^{*}$ be a given $C^{2}$ Jordan curve and $\lambda>0$. Suppose $\Omega, \Gamma, u$ is a solution of either free boundary problem with the following properties:

(i) $\Omega$ is a bounded, $C^{2}$ annular domain.

(ii) $u \in C^{2}(\overline{\mathbf{\Omega}})$.

Let $\left(x_{1}, y_{1}\right), \ldots,\left(x_{n}, y_{n}\right)$ be a positively ordered set of distinct points on $\Gamma$ (i.e. $\left(x_{1}, y_{1}\right)<\left(x_{2}, y_{2}\right)<\cdots<\left(x_{n}, y_{n}\right)<\left(x_{1}, y_{1}\right)$ in the natural ordering on $\Gamma$ ) such that for each $i$, the points $\left(x_{i}, y_{i}\right)$ has one of the following properties:

(a) For given unit vector $\vec{\nu}_{i}, \vec{n}\left(x_{i}, y_{i}\right)=\vec{\nu}_{i}$.

(b) $\vec{n}\left(x_{i}, y_{i}\right)=\vec{\nu}_{i}$ and $\left(x_{i}, y_{i}\right)$ is a $\vec{\nu}$-maximum ( $\vec{\nu}$-minimum) of $\Gamma$.

(c) $\left(x_{i}, y_{i}\right)$ is a positive (negative) inflection point of $\Gamma$.

Then there is a positively ordered set of distinct (possibly degenerate) line segments $\sigma_{1}, \ldots, \sigma_{n}$ on $\Gamma^{*}$ such that for each $i$, each point $(x, y)$ in the segment $\sigma_{i}$ has the same property relative to $\Gamma^{*}$ that $\left(x_{i}, y_{i}\right)$ has relative to $\Gamma$ (e.g. $\vec{n}(x, y)=\vec{\nu}_{i}$ for each $(x, y) \in \sigma_{i}$.) Further, the total positive (negative) curvature of $\Gamma$ is less than or equal to that of $\Gamma^{*}$.

When $\Gamma, \Gamma^{*}$, and $u$ are real-analytic and $\Omega$ is an annular domain, we obtain:

THEOREM 2. Let $\Gamma^{*}$ be a given analytic Jordan curve and $\lambda>0$. Suppose $\Omega, \Gamma, u$ is a solution of either free boundary problem with the following properties:

(i) $\Omega$ is a bounded, analytic annular domain.

(ii) $u$ is real-analytic on $\bar{\Omega}$.

Let $\vec{\nu}$ be a unit vector and let $\left(x_{1}, y_{1}\right), \ldots,\left(x_{n}, y_{n}\right)$ be the distinct $\vec{\nu}$-minima ( $\vec{\nu}$-maxima) of $\Gamma$. Then each point $\left(x_{i}, y_{i}\right)$ is joined by a simple, piecewise-analytic directed curve $\gamma_{i}$ to a point $\left(x_{i}^{*}, y_{i}^{*}\right) \in \Gamma^{*}$ such that

(a) $|\nabla u|$ is strictly increasing (decreasing) on $\gamma_{i}$.

(b) $\arg (\nabla u)$ is constant on $\gamma_{i}$.

(c) The points $\left(x_{1}^{*}, y_{1}^{*}\right), \ldots,\left(x_{n}^{*}, y_{n}^{*}\right)$ are distinct $\vec{\nu}$-minima $(\vec{\nu}$ maxima) of $\Gamma^{*}$. 
(d) $\left|\nabla u\left(x_{i}^{*}, y_{i}^{*}\right)\right|>(<) \lambda$ for each $i$.

(e) For $i \neq j$, the directed curves $\gamma_{i}$ and $\gamma_{j}$ do not cross or coalesce. Further, the total positive (negative) curvature of $\Gamma$ is less than or equal to that of $\Gamma^{*}$.

When $\Omega$ is a bounded domain which is not annular, we obtain the following two results.

THEOREM 3. Let $\Gamma^{*}$ be a given finite union of pairwise disjoint, $C^{2}$ Jordan curves and $\lambda>0$. Suppose $\Omega, \Gamma, u$ is a solution of either free boundary problem with the following properties:

(i) $\Omega$ is a bounded $C^{2}$ domain.

(ii) $u \in C^{2}(\overline{\mathbf{\Omega}})$.

Let $\left(x_{1}, y_{1}\right), \ldots,\left(x_{n}, y_{n}\right)$ be a positively ordered set of distinct $\vec{\nu}$ minima of $\Gamma$. Then there is a positively ordered set of distinct (possibly degenerate) line segments $\sigma_{1}, \ldots, \sigma_{n}$ on $\Gamma^{*}$ such that for each $i$, each point $(x, y)$ in the segment $\sigma_{i}$ is a $\vec{\nu}$-minimum of $\Gamma^{*}$. Further, the total positive curvature of $\Gamma$ is less than or equal to that of $\Gamma^{*}$.

THeOREM 4. Let $\Gamma^{*}$ be a given finite union of pairwise disjoint, analytic Jordan curves and $\lambda>0$. Suppose $\Omega, \Gamma, u$ is a solution of either free boundary problem with the following properties:

(i) $\Omega$ is a bounded, analytic domain.

(ii) $u$ is real-analytic on $\bar{\Omega}$.

Let $\vec{\nu}$ be a unit vector and let $\left(x_{1}, y_{1}\right), \ldots,\left(x_{n}, y_{n}\right)$ be the distinct $\vec{\nu}$ minima of $\Gamma$. Then each point $\left(x_{i}, y_{i}\right)$ is joined by a simple, piecewiseanalytic directed curve $\gamma_{i}$ to a point $\left(x_{i}^{*}, y_{i}^{*}\right) \in \Gamma^{*}$ such that

(a) $|\nabla u|$ is strictly increasing on $\gamma_{i}$.

(b) $\arg (\nabla u)$ is constant on $\gamma_{i}$. $\Gamma^{*}$.

(c) The points $\left(x_{1}^{*}, y_{1}^{*}\right), \ldots,\left(x_{n}^{*}, y_{n}^{*}\right)$ are distinct $\vec{\nu}$-minima of

(d) $\left|\nabla u\left(x_{i}^{*}, y_{i}^{*}\right)\right|>\lambda$ for each $i$.

(e) For $i \neq j$, the directed curves $\gamma_{i}$ and $\gamma_{j}$ do not cross or coalesce. Further, the total positive curvature of $\Gamma$ is less than or equal to that of $\Gamma^{*}$.

2. Preliminary results. Let us define $e(x, y) \equiv u_{x x}(x, y) u_{y y}(x, y)-$ $u_{x y}(x, y)^{2}, E=\{(x, y) \in \bar{\Omega}: e(x, y)<0\}$, and $Z=\{(x, y) \in \bar{\Omega}$ : $|\nabla u(x, y)|>0\}$. Further, set

$$
\phi(x, y)=|\nabla u(x, y)|^{2}, \quad(x, y) \in \bar{\Omega},
$$


and

$$
\psi(x, y)=\arg (\nabla u(x, y)), \quad(x, y) \in \bar{\Omega} \backslash Z .
$$

Notice that $\psi$ is a multiple-valued function. If $\alpha \in \mathfrak{R}, \vec{\eta}=(\sin (\alpha)$, $-\cos (\alpha))$, and $\vec{\eta}^{\perp}=(\cos (\alpha), \sin (\alpha))$, then $\{(x, y) \in \bar{\Omega} \backslash Z$ : $\psi(x, y)=\alpha\}=\left\{(x, y) \in \bar{\Omega}: \vec{\eta} \cdot \nabla u(x, y)=0, \vec{\eta}^{\perp} \cdot \nabla u(x, y)>0\right\}$.

The following lemma indicates the behavior of $\phi$ on level sets of $\psi$ when the graph of $u$ is a saddle surface.

LemMA 1. Suppose $u \in C^{2}(\Omega)$ satisfies $u_{x x} u_{y y}-u_{x y}^{2} \leq 0$ on $\Omega$. For $\alpha \in \Re$, define

$$
S_{\alpha}=\{(x, y) \in \Omega: \psi(x, y)=\alpha\} .
$$

Suppose $\left(x_{0}, y_{0}\right) \in S_{\alpha} \cap E \cap Z$. Then locally near $\left(x_{0}, y_{0}\right)$, the set $S_{\alpha}$ is a simple, $C^{1}$ curve $\sigma$ which divides its complement into two connected components on which $\psi-\alpha$ has opposite signs. Further, $\phi$ is strictly increasing on $\sigma$ if we choose the forward direction such that $\psi>\alpha$ locally to the right of $\sigma($ or $\psi<\alpha$ locally to the left of $\sigma)$.

Proof. Notice that $\nabla \psi=|\nabla u|^{-2}\left(u_{x} u_{x y}-u_{y} u_{x x}, u_{x} u_{y y}-u_{y} u_{x y}\right)$ and $\nabla \phi=2\left(u_{x} u_{x x}+u_{y} u_{x y}, u_{x} u_{x y}+u_{y} u_{y y}\right)$. Now $|\nabla \psi(x, y)|=0$ iff $e(x, y)=0$ iff $(x, y) \notin E$. Since $\left(x_{0}, y_{0}\right) \in E,\left|\nabla \psi\left(x_{0}, y_{0}\right)\right| \neq 0$. The first part now follows from the implicit function theorem. Let us now orient $\sigma$ so that $\psi>\alpha$ locally to the right of $\sigma$. Let us set $\nabla \psi^{\perp}=\left(-\psi_{y}, \psi_{x}\right)$. Notice that $\nabla \psi$ is orthogonal to $\sigma$ and points to the right of $\sigma$. Also, $\nabla \psi^{\perp}$ is a (forward) tangent vector to $\sigma$. Let us write

$$
\nabla \phi(x, y)=\beta_{1}(x, y) \nabla \psi(x, y)+\beta_{2}(x, y) \nabla \psi^{\perp}(x, y),
$$

where $\beta_{2}(x, y)=\nabla \phi(x, y) \cdot \nabla \psi^{\perp}(x, y) /|\nabla \psi(x, y)|^{2}$. We claim that $\beta(x, y)>0$ for all $(x, y) \in \sigma$. In fact, a direct computation yields

$$
\nabla \phi \cdot \nabla \psi^{\perp}=2\left(u_{x y}^{2}-u_{x x} u_{y y}\right)>0
$$

on $\sigma$ and our claim follows. If we parametrize $\sigma$ by $(x(t), y(t))$, then

$$
\nabla \phi \cdot\left(x^{\prime}, y^{\prime}\right)=\beta_{2}\left(\nabla \psi^{\perp} \cdot\left(x^{\prime}, y^{\prime}\right)\right)>0
$$

and so $\phi(x(t), y(t))$ is strictly increasing in $t$. 
Corollary. Suppose $u \in C^{2}(\bar{\Omega})$ satisfies $u_{x x} u_{y y}-u_{x y}^{2} \leq 0$ on $\Omega$, (2b), and (2c) and suppose $\sigma$ is an open line segment with $\sigma \subseteq \partial \Omega$ such that $\sigma \cap E$ is dense in $\sigma$. Then $\phi$ is strictly decreasing on $\sigma$ and $\psi \leq \alpha$ locally to the right of $\sigma$.

Here we orient $\sigma$ as a subset of $\partial \Omega$; that is, $\Omega$ is locally to the left of $\sigma$ when $\sigma \subseteq \Gamma$ and locally to the right of $\sigma$ when $\sigma \subset \Gamma^{*}$.

Proof. On $\sigma$, notice that $\nabla \psi$ is orthogonal to $\sigma$ and $\nabla \psi^{\perp}$ points backwards (or to the left) along $\sigma$. The fact that $\phi$ is strictly decreasing on $\sigma$ follows from the proof of Lemma 1 . Now if $\psi>\alpha$ locally to the right of a point $z$ of $\sigma$, then Lemma 1 would imply that $\phi$ is strictly increasing on $\sigma$ near $z$, a contradiction. Thus $\psi \leq \alpha$ in a neighborhood in $\Omega$ of $\sigma$.

REMARK. When $u \in C^{2}(\Omega)$ is a solution of $\left(2 \mathrm{a}^{\prime}\right)$, we may regard $u$ as a solution of the quasilinear equation (2a) for some $Q$ as in [12, p. 444]. If we set

$$
\begin{aligned}
& F_{i j}\left(x, y, p, q, r_{11}, r_{12}, r_{21}, r_{22}\right) \\
& \quad=\frac{\partial F}{\partial r_{i j}}\left(x, y, p, q, r_{11}, r_{12}, r_{21}, r_{22}\right),
\end{aligned}
$$

for $i, j=1,2$ (with $r_{12}=r_{21}$ ), then we see that $u$ is a solution of (2a) when $a=a^{11}, b=a^{12}$, and $c=a^{22}$ and

$$
a^{i j}(x, y, p, q)=\int_{0}^{1} F_{i j}\left(x, y, p, q, \theta D^{2} u(x, y)\right) d \theta .
$$

Thus, in the proofs throughout this note we will only consider solutions of $(2 \mathrm{a})$ (e.g. [12, §17.1]).

Lemma 2. Suppose $u \in C^{2}(\Omega)$ satisfies either (2a) or $\left(2 a^{\prime}\right)$. Then $u_{x x} u_{y y}-u_{x y}^{2} \leq 0$ in $\Omega$ and $u_{x x} u_{y y}-u_{x y}^{2}=0$ at a point if and only if $u_{x x}=u_{x y}=u_{y y}=0$ at the point. Further, if $D^{2} u \not \equiv 0$, then $E \cap \Omega$ and $Z \cap \Omega$ are open, dense subsets of $\Omega$ and if $\eta_{1}, \eta_{2} \in \mathfrak{R}$ with $\eta_{1}^{2}+\eta_{2}^{2}>0$ and $\sigma=\left\{(x, y) \in \Omega: \eta_{1} u_{x}(x, y)+\eta_{2} u_{y}(x, y)=0\right\}$, then $\sigma \cap E$ is dense in $\sigma$. If $u \in C^{2}(\bar{\Omega})$ and $\sigma=\left\{(x, y) \in \bar{\Omega}: \eta_{1} u_{x}(x, y)+\right.$ $\left.\eta_{2} u_{y}(x, y)=0\right\}$, then $\sigma \cap E$ is also dense in $\sigma$.

Proof. We may assume $u$ satisfies (2a). If we set $r=u_{x x}, s=u_{x y}$, and $t=u_{y y}$, we see that $0=r(a r+2 b s+c t)$ and so $r t-s^{2}=$ $-\frac{1}{c}\left(a r^{2} 2 b r s+c s^{2}\right)$. Since $Q$ is elliptic, $\alpha \xi_{1}^{2}+2 b \xi_{1} \xi_{2}+c \xi_{2}^{2}>0$ if and 
only if $\vec{\xi}=\left(\xi_{1}, \xi_{2}\right) \neq 0$. Thus $u_{x x} u_{y y}-u_{x y}^{2} \leq 0$. The fact that $E$ and $Z$ are dense follows from the strong maximum principle and the density of $\sigma \cap E$ in $\sigma$ follows from the proof of Lemma 1 of [13] after we rotate $\Omega$ so that $\vec{\eta}$ becomes $(0,1)$.

REMARK. Suppose $\Omega$ is a $C^{2}$ domain and $u \in C^{2}(\Omega \cup \Gamma) \cap C^{1}(\bar{\Omega})$. Then $|\nabla u| \neq 0$ on $\partial \Omega$ and $\Gamma$ cannot contain any line segments. Notice that $|\nabla u|$ is bounded in $\bar{\Omega}$ and so $Q$ or $\mathfrak{I}$ is uniformly elliptic for $u$. The first claim follows from the Hopf boundary point lemma (Lemma 3.4, [12]) and the second follows from the corollary to Lemma 1.

A. Annular domains. Here we will assume the following:

(i) $\Gamma^{*}$ is a given $C^{2}$ Jordan curve and $\lambda>0$.

(ii) $\Omega, \Gamma, u$ constitutes a solution of one of the free boundary problems.

(iii) $\Omega$ is an annular domain and $\Gamma$ is a $C^{2}$ Jordan curve.

(iv) $u \in C^{2}(\Omega) \cap C^{1}(\bar{\Omega})$.

LEMMA 3. For each $(x, y) \in \bar{\Omega},|\nabla u(x, y)|>0$ and $Z=\bar{\Omega}$.

Proof. Let $\left(x_{0}, y_{0}\right) \in E$ and define $\tilde{u}$ by

$$
\tilde{u}(x, y)=z_{0}+p_{0}\left(x-x_{0}\right)+q_{0}\left(y-y_{0}\right),
$$

where $z_{0}=u\left(x_{0}, y_{0}\right)>0, p_{0}=u_{x}\left(x_{0}, y_{0}\right)$, and $q_{0}=u_{y}\left(x_{0}, y_{0}\right)$. As in Lemma 2, [13], we see that $\tilde{u} \equiv u$ or there are two curves $\sigma_{1}, \sigma_{2}$ which meet at $\left(x_{0}, y_{0}\right)$ and divide a neighborhood of $\left(x_{0}, y_{0}\right)$ into four open "sectors" $\omega_{1}, \omega_{2}, \omega_{3}, \omega_{4}$ such that $u>\tilde{u}$ in $\omega_{2} \cup \omega_{3}$ and $u<\tilde{u}$ in $\omega_{2} \cup \omega_{4}$. If the plane $z=\tilde{u}$ does not intersect $\Sigma_{0} \cup \Sigma_{1}$, where $\Sigma_{0}=\Gamma \times\{0\}$ and $\Sigma_{1}=\Gamma^{*} \times\{1\}$, then $\tilde{u}>0=u$ on $\Gamma$ and $\tilde{u}<1=u$ on $\Gamma^{*}$. Since $\Omega$ is an annular domain, the previous two statements are in contradiction; this follows, for example, from the maximum principle and the Jordan curve theorem (e.g. the proof in $\S 373,[16])$. Hence, the tangent plane to the graph of $u$ at $\left(x_{0}, y_{0}\right)$ must intersect $\Sigma_{0} \cup \Sigma_{1}$. This implies that

$$
\begin{aligned}
& \left|\nabla u\left(x_{0}, y_{0}\right)\right| \\
& \quad \geq \min \left\{u\left(x_{0}, y_{0}\right) d\left(x_{0}, y_{0}\right),\left(1-u\left(x_{0}, y_{0}\right)\right) d^{*}\left(x_{0}, y_{0}\right)\right\}>0
\end{aligned}
$$

for every $\left(x_{0}, y_{0}\right) \in E$, where $d(x, y)=\inf \left\{|(x, y)-(s, t)|^{-1}\right.$ : $(s, t) \in \Gamma\}$ and $d^{*}(x, y)=\inf \left\{|(x, y)-(s, t)|^{-1}:(s, t) \in \Gamma^{*}\right\}$. Since 
$u \in C^{2}(\Omega)$, (4) holds for all $\left(x_{0}, y_{0}\right) \in \Omega$. Since we already know $|\nabla u|>0$ on $\partial \Omega$, the lemma follows.

LEMMA 4. Let $\left(x_{0}, y_{0}\right) \in \Gamma$ and let $\vec{\eta}=\left(\eta_{1}, \eta_{2}\right)$ with $|\eta|=1$ and $\vec{\eta} \cdot \nabla u\left(x_{0}, y_{0}\right) \neq 0$. Let $\omega$ denote the connected component of $\{(x, y) \in \bar{\Omega}: \vec{\eta} \cdot \nabla u(x, y) \neq 0\}$ which contains $\left(x_{0}, y_{0}\right)$. Let $\alpha \in \mathfrak{R}$ such that $\vec{\eta}=(\sin (\alpha),-\cos (\alpha))$. Then:

(a) $\omega$ is relatively open in $\bar{\Omega}$ and $\omega \cap \Gamma^{*}$ is relatively open in $\Gamma^{*}$.

(b) $\omega$ is simply connected and $\omega \cap \Gamma$ is connected.

(c) $\vec{\eta} \cdot \nabla u=0$ on $\Omega \cap \partial \omega$ and $\psi$ is constant $(=\alpha$ or $\alpha+\pi$ $(\bmod 2 \pi)$ ) on each component of $\Omega \cap \partial \omega$.

(d) $\omega \cap \Gamma^{*} \neq \varnothing$.

(e) $\partial \omega$ is a simple, $C^{1}$ curve in a neighborhood of each point of $\partial \omega \cap E$.

(f) If a component $\gamma$ of $\Omega \cap \partial \omega$ is oriented so that $\vec{\eta} \cdot \nabla u<0$ locally to the right or $\vec{\eta} \cdot \nabla u>0$ locally to the left, then $\phi$ is strictly increasing on $\bar{\gamma}$.

Proof. Notice that (a), (c), and (e) are clear. If $\gamma$ is a component of $\partial \omega \cap \Omega$, then $\gamma \cap E$ is dense in $\gamma$ and $\psi=\theta$ with $\theta=\alpha$ or $\theta=\alpha+\pi \bmod 2 \pi$. Suppose $\gamma$ is oriented as in (f). Then $\psi>\theta$ locally to the right or $\psi<\theta$ locally to the left and so $\phi$ is strictly increasing on $\gamma \cap E$ by Lemma 1. Since $\phi \in C^{0}(\bar{\Omega})$ and $\gamma \cap E$ is relatively open and dense in $\gamma$, (f) follows. Suppose $\omega$ is not simply connected. Then there is a component $\gamma$ of $\partial \omega$ and a bounded component $U$ of $\Re \backslash \omega$ such that $\gamma=\partial U$. If $\gamma \subseteq \Omega$, then the strict monotonicity of $\phi$ yields a contradiction unless $U$ is a single point, in which case the strong maximum principle implies $\vec{\eta} \cdot \nabla u \neq 0$ in $U$ and so $U \subset \omega$, a contradiction. Thus $\gamma \cap \partial \Omega \neq \varnothing$. For convenience, let us assume $\vec{\eta} \cdot \nabla u>0$ in $\omega$. The monotonicity of $\phi$ implies $\gamma \cap \Gamma$ contains no more than one point and $U$ must contain a component $\Gamma_{0}^{*}$ of $\Gamma^{*}$. Let $\left(x_{0}, y_{0}\right) \in \Gamma$ with $\vec{\eta} \cdot \nabla u\left(x_{0}, y_{0}\right)<0$ and let $\omega_{0}$ be the component of $W$ which contains $\left(x_{0}, y_{0}\right)$. Then $\omega \cap \omega_{0}=\varnothing$ and so $\omega_{0} \cap \Gamma_{0}^{*}=\varnothing$. If $\Gamma^{*}$ contains only one component, this contradicts $(\mathrm{d})$. Suppose next that $\omega \cap \Gamma$ is not connected. Then there is a component $\gamma$ of $\partial \omega \cap \Omega$ which joins two points of $\Gamma$. Since $\vec{\eta} \cdot \nabla u=0$ on $\gamma$, $\phi$ is strictly monotonic on $\gamma$. However, this contradicts the fact that $\phi=\lambda^{2}$ on $\Gamma$; hence (b) holds. Finally suppose $\omega \cap \Gamma^{*}=\varnothing$. Then $\partial \omega$ is a Jordan curve in $\Omega \cup \Gamma$. Now $\vec{\eta} \cdot \nabla u=0$ on $\partial \omega \cap \Omega$ and so $\phi$ is strictly monotonic on $\partial \omega \cap \Omega$. Once again, this contradicts $\phi=\lambda^{2}$ on $\Gamma$ and so (d) follows. 
LeMmA 5. Let $\omega_{1}, \omega_{2}$ be connected components of $\{(x, y) \in \bar{\Omega}$ : $\vec{\eta} \cdot \nabla u(x, y) \neq 0\}$ for some $\vec{\eta}$ with $|\vec{\eta}|=1$. If $\left(\omega_{1} \cup \omega_{2}\right) \cap \Gamma$ is not connected, then $\omega_{1} \cap \omega_{2}=\varnothing$.

Proof. If $\omega_{1} \cap \omega_{2} \neq \varnothing$, then $\omega_{1}=\omega_{2}$ and so $\left(\omega_{1} \cup \omega_{2}\right) \cap \Gamma=\omega_{1} \cap \Gamma$ is connected by (b) of Lemma 3.

B. General domains. Here we will assume the following:

(i) $\Gamma^{*}$ is a given finite union of disjoint $C^{2}$ Jordan curves and $\lambda>0$.

(ii) $\Omega, \Gamma, u$ constitutes a solution of one of the free boundary problems.

(iii) $\Omega$ is a bounded domain and $\Gamma$ is a finite union of disjoint $C^{2}$ Jordan curves.

(iv) $u \in C^{2}(\Omega) \cap C^{1}(\bar{\Omega})$.

In this case, we expect $|\nabla u|=0$ at some points.

LeMmA 6. Let $\left(x_{0}, y_{0}\right) \in \Gamma$ and let $\vec{\eta}=\left(\eta_{1}, \eta_{2}\right)$ with $|\eta|=1$ and $\vec{\eta} \cdot \nabla u\left(x_{0}, y_{0}\right) \neq 0$. Let $\omega$ denote the connected component of $\{(x, y) \in \bar{\Omega}: \vec{\eta} \cdot \nabla u(x, y) \neq 0\}$ which contains $\left(x_{0}, y_{0}\right)$. Let $\alpha \in \mathfrak{R}$ such that $\vec{\eta}=(\sin (\alpha),-\cos (\alpha))$. Then:

(a) $\omega$ is relatively open in $\bar{\Omega}$ and $\omega \cap \Gamma^{*}$ is relatively open in $\Gamma^{*}$.

(b) $\omega \cap \Gamma$ is connected and if $\omega$ is not simply connected and if $U$ is a bounded component of $\mathfrak{R}^{2} \backslash \omega$, then $U$ contains a component $\Gamma_{0}^{*}$ of $\Gamma^{*}$.

(c) $\bar{\eta} \cdot \nabla u=0$ on $\Omega \cap \partial \omega$ and $\psi$ is constant $(=\alpha$ or $\alpha+\pi$ $(\bmod 2 \pi))$ on each component of $Z \cap \partial \omega$.

(d) $\omega \cap \Gamma^{*} \neq \varnothing$.

(e) $\partial \omega$ is a simple, $C^{1}$ curve in a neighborhood of each point of $\partial \omega \cap E \cap Z$.

(f) If a component $\gamma$ of $Z \cap \partial \omega$ is oriented so that $\vec{\eta} \cdot \nabla u<0$ locally to the right or $\vec{\eta} \cdot \nabla u>0$ locally to the left, then $\phi$ is strictly increasing on $\bar{\gamma}$.

The proof is essentially the same as the proof of Lemma 4. Notice that $|\nabla u| \neq 0$ in $\omega$.

LEMMA 7. Let $\omega_{1}, \omega_{2}$ be connected components of $\{(x, y) \in \bar{\Omega}$ : $\vec{\eta} \cdot \nabla u(x, y) \neq 0\}$ for some $\vec{\eta}$ with $|\vec{\eta}|=1$. If $\left(\omega_{1} \cup \omega_{2}\right) \cap \Gamma$ is not connected, then $\omega_{1} \cap \omega_{2}=\varnothing$.

The proof is the same as the proof of Lemma 5. 
C. The real analytic case. Here we will assume the following:

(i) $\Gamma^{*}$ is a given finite union of disjoint real-analytic Jordan curves and $\lambda>0$.

(ii) $\Omega$ is a bounded domain and $\Gamma$ is a finite union of disjoint real-analytic Jordan curves.

(iii) $u$ is real-analytic on $\bar{\Omega}$ and either $Q$ is real-analytic on $\mathfrak{R}^{2} \times \mathfrak{R}^{2}$ or $\mathfrak{I}$ is real-analytic on $\mathfrak{R}^{2} \times \mathfrak{R}^{2} \times \mathfrak{R}^{2 \times 2}$.

For each unit vector $\vec{\nu}=v_{1} \vec{i}+\nu_{2} \vec{j}$, let $H_{\vec{\nu}}$ denote the set of piecewise analytic, directed curves $\gamma$ in $\bar{\Omega}$ on which $|\nabla u|$ is strictly increasing and $\nabla u(x, y)$ points in the $\vec{\nu}$-direction at each point $(x, y)$ of $\gamma$. Since $u$ is real-analytic on $\bar{\Omega}$, we wish to regard $u$ as a realanalytic solution of $(2 \mathrm{a})$ in a neighborhood of $\bar{\Omega}$. This will simplify the statement of certain results (e.g. Lemma 9).

Lemma 8. Suppose $\left(x_{0}, y_{0}\right) \in \Omega$. Set

$$
\tilde{u}(x, y)=z_{0}+p_{0}\left(x-x_{0}\right)+q_{0}\left(y-y_{0}\right)
$$

where $z_{0}=u\left(x_{0}, y_{0}\right) \in(0,1), p_{0}=u_{x}\left(x_{0}, y_{0}\right)$, and $q_{0}=u_{y}\left(x_{0}, y_{0}\right)$. Then either $u \equiv \tilde{u}$ or

$$
u(x, y)=\tilde{u}(x, y)+H(\bar{x}, \bar{y})+O\left(r^{n+1}\right) \quad \text { as } r \rightarrow 0,
$$

where $n \geq 2$ is an integer, $r=\operatorname{dist}\left((x, y),\left(x_{0}, y_{0}\right)\right), H$ is a harmonic, homogeneous polynomial of degree $n$ in $(\bar{x}, \bar{y})$, and $\bar{x}, \bar{y}$ are linearly independent, linear functions of $x, y$.

Let $\eta_{1}, \eta_{2} \in \mathfrak{R}$ with $\eta_{1}^{2}+\eta_{2}^{2}=1$. Suppose $u \not \equiv \tilde{u}, \eta_{1} u_{x}\left(x_{0}, y_{0}\right)+$ $\eta_{2} u_{y}\left(x_{0}, y_{0}\right)=0$, and $\left(x_{0}, y_{0}\right)$ is a branch point of $u$ (i.e. $u_{x x}\left(x_{0}, y_{0}\right)$ $\left.=u_{x y}\left(x_{0}, y_{0}\right)=u_{y y}\left(x_{0}, y_{0}\right)=0\right)$. Then $n \geq 3$ and

$$
\eta_{1} u_{x}(x, y)+\eta_{2} u_{y}(x, y)=G(\bar{x}, \bar{y})+O\left(r^{n}\right) \quad \text { as } r \rightarrow 0,
$$

where $G$ is a harmonic, homogeneous polynomial of degree $n-1$. Hence the zeros of $\eta_{1} u_{x}+\eta_{2} u_{y}$ in a neighborhood of $\left(x_{0}, y_{0}\right)$ lie on $n-1 \geq 2$ analytic curves which intersect at $\left(x_{0}, y_{0}\right)$ and divide a neighborhood of $\left(x_{0}, y_{0}\right)$ into $2(n-1)$ disjoint open "sectors" $\omega_{1}, \ldots, \omega_{2 n-2}$ such that $\eta_{1} u_{x}+\eta_{2} u_{y}<0$ in $\omega_{1}, \ldots, \omega_{2 n-3}$ and $\eta_{1} u_{x}+\eta_{2} u_{y}>0$ in $\omega_{2}, \ldots, \omega_{2 n-2}$.

Proof. The proof of the first part is similar to [19, p. 380]; also see [13]. Let us consider the second part. Suppose $\bar{x}=\varepsilon_{1}\left(x-x_{0}\right)+$ $\varepsilon_{2}\left(y-y_{0}\right)$ and $\bar{y}=\delta_{1}\left(x-x_{0}\right)+\delta_{2}\left(y-y_{0}\right)$; here, $\varepsilon_{1} \delta-\varepsilon_{2} \delta_{1} \neq 0$. Now $\frac{\partial H}{\partial x}(\bar{x}, \bar{y})=\varepsilon_{1} H_{\bar{x}}(\bar{x}, \bar{y})+\delta_{1} H_{\bar{y}}(\bar{x}, \bar{y})$ and $\frac{\partial H}{\partial y}(\bar{x}, \bar{y})=\varepsilon_{2} H_{\bar{x}}(\bar{x}, \bar{y})+$ 
$\delta_{2} H_{\bar{y}}(\bar{x}, \bar{y})$. Since $\eta_{1} p_{0}+\eta_{2} q_{0}=0$, we obtain (14) when $G(\bar{x}, \bar{y})=$ $\left(\eta_{1} \varepsilon_{1}+\eta_{2} \varepsilon_{2}\right) H_{\bar{x}}(\bar{x}, \bar{y})+\left(\eta_{1} \delta_{1}+\eta_{2} \delta_{2}\right) H_{\bar{y}}(\bar{x}, \bar{y})$. Since $H_{\bar{x}}$ and $H_{\bar{y}}$ are harmonic, homogeneous polynomials of degree $n-1$, either $G \equiv 0$ or $G$ is as claimed. Since $u \not \equiv \tilde{u}, H_{\bar{x}}$ and $H_{\bar{y}}$ are linearly independent; it then follows that $G \not \equiv 0$. The remainder of the proof follows using standard facts about harmonic functions (e.g. [16], §373).

Lemma 9. For $\alpha \in \mathfrak{R}$, define

$$
S_{\alpha}=\{(x, y) \in Z \cup \partial \Omega: \psi(x, y)=\alpha\} .
$$

Then

(a) If $\left|\nabla \psi\left(x_{0}, y_{0}\right)\right| \neq 0$ at a point $\left(x_{0}, y_{0}\right) \in S_{\alpha}$, then locally at $\left(x_{0}, y_{0}\right)$ the set $S_{\alpha}$ is an analytic simple curve $\gamma$ which divides its complement into two connected components on which $\psi-\alpha$ has opposite signs. Further, $\phi$ is strictly increasing on $\gamma$ if we choose the forward direction such that $\psi>\alpha$ locally to the right of $\gamma$.

(b) Suppose $\nabla \psi$ has a zero of order $n$ at $\left(x_{0}, y_{0}\right) \in S_{\alpha}$, for some integer $n \geq 1$. Then locally at $\left(x_{0}, y_{0}\right)$, the set $S_{\alpha}$ is swept out by $2 n+2$ directed, analytic arcs $C_{1}, C_{2}, \ldots, C_{2 n+2}$ which emanate from $\left(x_{0}, y_{0}\right)$ and divide its complement into $2 n+2$ open sectors

$$
\omega_{1}, \omega_{2}, \ldots, \omega_{2 n+2}
$$

on which $\psi-\alpha$ alternates in sign. We may choose our notation so that $C_{i} \cup C_{i+1}$ is the local boundary of $\omega_{i}$ (with $\left.C_{2 n+3}=C_{1}\right),(-1)^{i}(\psi-\alpha)$ is negative in $\omega_{i}$, and $(-1)^{i} \phi$ is strictly decreasing on $C_{i}$.

Proof. Notice that (a) follows Lemma 1. If we set $\vec{\eta}=(\sin (\alpha)$, $-\cos (\alpha))$ and $\vec{\eta}^{\perp}=(\cos (\alpha), \sin (\alpha))$, then $S_{\alpha}=\{(x, y) \in \bar{\Omega}: \vec{\eta}$. $\left.\nabla u(x, y)=0, \vec{\eta}^{\perp} \cdot \nabla u(x, y)>0\right\}$. Suppose $\left(x_{0}, y_{0}\right) \in S_{\alpha}$ such that $\left|\nabla u\left(x_{0}, y_{0}\right)\right|=0$. Notice that $\left(x_{0}, y_{0}\right) \notin E$. According to the second part of Lemma 8 , we see that near $\left(x_{0}, y_{0}\right)$ the set $\vec{\eta} \cdot \nabla u=0$ consists of $n-1 \geq 2$ analytic curves through $\left(x_{0}, y_{0}\right)$. Since $S_{\alpha}$ is contained in the relatively open set $\left\{(x, y) \in \bar{\Omega}: \vec{\eta}^{\perp} \cdot \nabla u(x, y)>0\right\}$, we see that the set $\psi=\alpha$ consists of the same $n-1$ analytic curves and the remainder of the lemma follows from Lemma 1 and Lemma 8.

LEMMA 10. Let $\vec{\nu}$ be a unit vector and let $\Gamma$ have a $\vec{\nu}$-minimum at $\left(x_{0}, y_{0}\right)$. Then:

(a) At least one directed curve $\tilde{\gamma} \in H_{\vec{\nu}}$, which exists locally at $\left(x_{0}, y_{0}\right)$, emanates into $\Omega$ from $\left(x_{0}, y_{0}\right)$. 
(b) Let $\gamma \in H_{\vec{\nu}}$ be any maximal continuation of $\tilde{\gamma}$ in $H_{\vec{\nu}}$. Then $\gamma$ remains uniformly bounded away from any zero of $|\nabla u|$ and any closed, connected set in $\Gamma^{*}$ on which $\vec{n}(x, y) \neq \vec{\nu}$ and $\gamma \backslash \tilde{\gamma}$ remains bounded away from $\Gamma$.

(c) The curve $\gamma$ must terminate at a point $\left(x_{0}^{*}, y_{0}^{*}\right) \in \Gamma^{*}$ such that

$$
\vec{n}\left(x_{0}^{*}, y_{0}^{*}\right)=\vec{\nu}
$$

and

$$
\left|\nabla u\left(x_{0}^{*}, y_{0}^{*}\right)\right|>\lambda
$$

REMARK. If $\Omega$ is an annular domain, then Lemma 3 implies $|\nabla u|>$ 0 on $\bar{\Omega}$ and the conclusions of Lemma 10 hold when $\Gamma$ has a $\vec{\nu}$ maximum at $\left(x_{0}, y_{0}\right)$ with the modifications that $\tilde{\gamma} \in H_{\vec{\nu}}$ terminates at $\left(x_{0}, y_{0}\right), \gamma$ begins at $\left(x_{0}^{*}, y_{0}^{*}\right)$, and $\left|\nabla u\left(x_{0}^{*}, y_{0}^{*}\right)\right|<\lambda$.

The proof of Lemma 10 follows from Lemma 9 as in the proof of Lemma 2, [4].

\section{Proof of main results.}

Proof of Theorem 1. Suppose first that $\left(x_{0}, y_{0}\right) \in \Gamma$ and $\vec{n}\left(x_{0}, y_{0}\right)=$ $\vec{\nu}=\left(\nu_{1}, \nu_{2}\right)$. Set $\vec{\eta}=-\vec{\nu}^{\perp}=\left(\nu_{2},-\nu_{1}\right)$ and $W=\{(x, y) \in \Omega: \vec{\eta}$. $\nabla u(x, y) \neq 0\}$. Since $\Gamma$ contains no line segments and $\nabla u \in C^{0}(\bar{\Omega})$, $\left(x_{0}, y_{0}\right) \in \partial W$. Let $\omega$ be a component of $W$ such that $\left(x_{0}, y_{0}\right) \in \partial \omega$ and let $\gamma$ be a component of $\partial \omega \cap \Omega$ with $\left(x_{0}, y_{0}\right) \in \bar{\gamma}$. From Lemma 4 , we see that $\bar{\gamma} \cap \Gamma^{*} \neq \varnothing$ and $\vec{\eta} \cdot \nabla u=0$ on $\bar{\gamma}$. Also $\vec{\eta}^{\perp} \cdot \nabla u>0$ on $\bar{\gamma}$ since $\vec{\eta}^{\perp} \cdot \nabla u\left(x_{0}, y_{0}\right)=\vec{\nu} \cdot \nabla u\left(x_{0}, y_{0}\right)>0$ and $|\nabla u| \neq 0$ on $\bar{\Omega}$. Thus if $(x, y) \in \bar{\gamma} \cap \Gamma^{*}, \vec{n}(x, y)=\vec{\nu}$. If $\sigma_{0}$ is the maximal line segment on $\Gamma^{*}$ which contains $\bar{\gamma} \cap \Gamma^{*}$, then $\vec{n}(x, y)=\vec{\nu}$ for all $(x, y) \in \sigma_{0}$.

Suppose next that $\left(x_{0}, y_{0}\right) \in \Gamma$ with $\vec{n}\left(x_{0}, y_{0}\right)=\vec{\nu}$ such that $\left(x_{0}, y_{0}\right)$ is a $\vec{\nu}$-minimum of $\Gamma$. Let us use the notation of the previous paragraph. Then $\vec{\nu} \cdot(x, y)>\vec{\nu} \cdot\left(x_{0}, y_{0}\right)$ for $(x, y) \in \Gamma \backslash\left\{\left(x_{0}, y_{0}\right)\right\}$ near $\left(x_{0}, y_{0}\right)$. Since $\Gamma$ is the 0 -level curve of $u$, this implies $\vec{\eta} \cdot \nabla u>$ 0 locally before $\left(x_{0}, y_{0}\right)$ on $\Gamma$ and $\vec{\eta} \cdot \nabla u<0$ locally after $\left(x_{0}, y_{0}\right)$ on $\Gamma$. Now let $\omega_{ \pm}$be the components of $W$ such that $\left(x_{0}, y_{0}\right)$ is the left (or initial) endpoint of $\overline{\omega_{+}} \cap \Gamma$ and is the right (or terminal) endpoint of $\overline{\omega_{-}} \cap \Gamma$. Then $\vec{\eta} \cdot \nabla u<0$ in $\omega_{+}$and $\vec{\eta} \cdot \nabla u>0$ in $\omega_{-}$. Let $\gamma_{+}$be the component of $\partial \omega_{+} \cap \Omega$ whose closure contains $\left(x_{0}, y_{0}\right)$ and let $\gamma_{-}$be the component of $\partial \omega_{-} \cap \Omega$ whose closure contains $\left(x_{0}, y_{0}\right)$. As above, $\overline{\gamma_{ \pm}} \cap \Gamma^{*} \neq \varnothing$ and if $(x, y) \in \overline{\gamma_{ \pm}} \cap \Gamma^{*}$, then $\vec{n}(x, y)=\vec{\nu}$. Notice that $\bar{\gamma} \cap \Gamma^{*}$ lies to the left (or before) 
$\overline{\gamma_{ \pm}} \cap \Gamma^{*}$. If $\sigma_{ \pm}$are the (possibly degenerate) closed line segments in $\Gamma^{*}$ such that $\bar{\gamma}_{+} \cap \Gamma^{*} \subset \sigma_{+}$and $\overline{\gamma_{-}} \cap \Gamma^{*} \subset \sigma_{-}$and if $z_{-}, z_{+} \in \Gamma^{*}$ are the left endpoint of $\sigma_{-}$and the right endpoint of $\sigma_{+}$respectively, then $\vec{n} \cdot \nabla u>0$ and $\psi<\alpha$ locally to the left of $z_{-}$on $\Gamma^{*}$ and $\vec{\eta} \cdot \nabla u<0$ and $\psi>\alpha$ locally to the right of $z_{+}$on $\Gamma^{*}$, where $\alpha \in \mathfrak{R}$ with $(\cos (\alpha), \sin (\alpha))=\vec{\nu}$. The fact that $\Gamma^{*}$ has a $\vec{\nu}$-minimum between $z_{-}$and $z_{+}$follows using an argument similar to that of [1] (in particular, see Lemma 1 and the proof of Theorem 1 in [1]). The case where $\left(x_{0}, y_{0}\right)$ is a $\vec{\nu}$-maximum of $\Gamma$ follows similarly.

Now suppose $\left(x_{0}, y_{0}\right) \in \Gamma$ with $\vec{n}\left(x_{0}, y_{0}\right)=\vec{\nu}$ and $\left(x_{0}, y_{0}\right)$ is a positive inflection point of $\Gamma$. Then if $\vec{\eta}=-\vec{\nu}^{\perp}$ as above, $\vec{\nu} \cdot \nabla u<0$ locally before and locally after $\left(x_{0}, y_{0}\right)$ on $\Gamma$. Let $W=\{(x, y) \in$ $\Omega: \vec{\eta} \cdot \nabla u(x, y)<0\}$ and let $\omega_{ \pm}$be the components of $W$ and $\gamma_{ \pm}$ be the components of $\partial \omega_{ \pm} \cap \Omega$ as in the previous paragraph. Then $\vec{\eta} \cdot \nabla u=0$ on $\gamma_{ \pm}$and $\phi$ is strictly increasing (decreasing) on $\overline{\gamma_{+}}\left(\overline{\gamma_{-}}\right)$ as $(x, y) \in \overline{\gamma_{ \pm}}$leaves $\left(x_{0}, y_{0}\right)$. This implies $\overline{\gamma_{-}} \cap \overline{\gamma_{+}}=\left\{\left(x_{0}, y_{0}\right)\right\}$. Let $z_{-}, z_{+} \in \Gamma^{*}$ be the leftmost point of $\bar{\gamma}_{-} \cap \Gamma^{*}$ and the rightmost point of $\overline{\gamma_{+}} \cap \Gamma^{*}$ respectively. Let $V$ be the open subset of $\Omega$ bounded by $\gamma_{-} \cup \gamma_{+}$and that portion of $\Gamma^{*}$ between $z_{-}$and $z_{+}$. Let us suppose that $\vec{\eta} \cdot \nabla u \leq 0$ in $V$; this will lead to a contradiction. Let $z \in$ $\partial V \cap E \subset \gamma_{-} \cup \gamma_{+}$and pick $\varepsilon>0$ so that $\vec{\eta} \cdot \nabla u \leq 0$ in $B(z, \varepsilon) \subset \Omega$. Now $\partial B(z, \varepsilon) \cap\left(\omega_{-} \cup \omega_{+}\right) \neq \varnothing$ and so $\vec{\eta} \cdot \nabla u<0$ on a portion of the boundary of $B(z, \varepsilon)$. If we rotate $\Omega$ so that $\vec{\eta}=(0,1)$ and so $\vec{\eta} \cdot \nabla u=u_{y}$ and if we notice that $u_{y}$ is the generalized solution of a linear, elliptic, homogeneous equation (e.g. [12], §13.2), we see that the strong maximum principle (e.g. [12], Theorem 8.19) implies $\vec{\eta} \cdot \nabla u<0$ in $B(z, \varepsilon)$. However, $\vec{\eta} \cdot \nabla u=0$ on $\gamma_{-} \cup \gamma_{+}$and $z \in \gamma_{-} \cup \gamma_{+}$. This contradiction implies $\vec{\eta} \cdot \nabla u>0$ for some points in $V$. Now let $D$ be a component of $\{(x, y) \in \Omega: \vec{\eta} \cdot \nabla u(x, y)>0\}$ such that $D \subset V$. Since $\phi$ is strictly monotonic on $\partial D \cap \Omega$, we see that $\bar{D} \cap \Gamma^{*} \neq \varnothing$ and $\bar{D} \cap \Gamma^{*}$ must contain a point $z_{0}$ at which $\vec{\eta} \cdot \nabla u>0$. Since $\vec{\eta} \cdot \nabla u<0$ locally to the left of (or before) $z_{-}$and locally to the right of (or after) $z_{+}$and $z_{0}$ lies between $z_{-}$and $z_{+}, \Gamma^{*}$ must have a positive inflection point between $z_{-}$and $z_{+}$.

Notice that the final conclusion of the theorem concerning the total positive and negative curvature follows from (a) in a manner similar to the proof of Theorem 2 of [4] for the positive curvature case. To complete the proof, we need only observe that if $\vec{n}\left(x_{1}, y_{1}\right)=$ $\vec{n}\left(x_{2}, y_{2}\right)=\vec{\nu}$ for $\left(x_{1}, y_{1}\right),\left(x_{2}, y_{2}\right) \in \Gamma$ with $\left(x_{1}, y_{1}\right) \neq\left(x_{2}, y_{2}\right)$ and if $\gamma_{i}$ is $\gamma, \gamma_{-}$, or $\gamma_{+}$as above with $\left(x_{0}, y_{0}\right)=\left(x_{i}, y_{i}\right)$, then 
$\overline{\gamma_{1}} \cap \overline{\gamma_{2}}=\varnothing$. To see this, let $\left(x^{0}, y^{0}\right) \in \Gamma$ lie between $\left(x_{1}, y_{1}\right)$ and $\left(x_{2}, y_{2}\right)$ such that $\vec{n}\left(x^{0}, y^{0}\right)=\vec{e} \neq \vec{\nu}$. Set $\vec{\delta}=-\vec{e}^{\perp}$ and $W^{0}=\{(x, y) \in \Omega: \vec{\delta} \cdot \nabla u(x, y) \neq 0\}$. Now let $\omega^{0}$ be a component of $W^{0}$ with $\left(x^{0}, y^{0}\right) \in \overline{\omega^{0}}$ and let $\gamma^{0}$ be a component of $\partial \omega^{0} \cap \Omega$ with $\left(x^{0}, y^{0}\right) \in \overline{\gamma^{0}}$. Then $\gamma^{0}$ is a curve from $\left(x^{0}, y^{0}\right)$ with $\overline{\gamma^{0}} \cap \Gamma^{*} \neq \varnothing$ and $\vec{\delta} \cdot \nabla u=0$ on $\gamma^{0}$. Since $|\nabla u| \neq 0$ in $\bar{\Omega}, \overline{\gamma^{0}}$ (strictly) separates $\overline{\gamma_{1}}$ and $\overline{\gamma_{2}}$.

Proof of Theorem 2. When the $\left(x_{i}, y_{i}\right)$ are $\vec{\nu}$-minima of $\Gamma$, the proof of the existence of curves $\gamma_{i} \in H_{\vec{\nu}}$ and $\vec{\nu}$-minima $\left(x_{i}^{*}, y_{i}^{*}\right) \in \Gamma^{*}$ as indicated in the theorem is essentially the same as the proof of Theorem 5 of [4] with Lemmas 9 and 10 taking the places of Lemmas 1 and 2 of [4]. Since $|\nabla u|>0$ in $\bar{\Omega}$ (Lemma 3), we may modify the proof in [4] when the $\left(x_{i}, y_{i}\right)$ are $\vec{\nu}$-maxima of $\Gamma$. In fact, if we modify the rules in [4] for continuation of curves $\psi=\alpha$ so as to keep $\psi<\alpha$ locally to the right except at negative inflection points of $\Gamma^{*}$, where we require $\psi>\alpha$ locally to the left, then the proof of the existence of curves $\gamma_{i}$ on which $\phi$ is decreasing and which begin at $\left(x_{i}, y_{i}\right)$ and end at $\vec{\nu}$-maxima $\left(x_{i}^{*}, y_{i}^{*}\right)$ of $\Gamma^{*}$ as in the theorem is similar to the proof in the $\vec{\nu}$-minima case. The last conclusion of Theorem 2 follows from Theorem 1.

Proof of Theorem 3. In [4], continuation rules for curves of constant gradient direction were developed using the analyticity of $u$ and $\Gamma^{*}$ and these curves, which began at $\vec{\nu}$-minima of $\Gamma$, were shown to terminate at $\vec{\nu}$-minima of $\Gamma^{*}$. Since we do not know the behavior of $u$ in a neighborhood of a point $z$ of $\Gamma^{*}$ at which $e(z)=0$, a considerable portion of this proof will involve technical details required to allow us to continue curves of constant gradient direction which have reached $\Gamma^{*}$.

Suppose $\left(x_{0}, y_{0}\right) \in \Gamma$ with $\vec{n}\left(x_{0}, y_{0}\right)=\vec{\nu}$ and $\left(x_{0}, y_{0}\right)$ is a $\vec{\nu}$ minimum of $\Gamma$ and set $\vec{\eta}=-\vec{\nu}^{\perp}$. Let $W_{+}=\{(x, y) \in \Omega: \vec{\eta}$. $\nabla u(x, y)<0\}$ and $W_{-}=\{(x, y) \in \Omega: \vec{\eta} \cdot \nabla u(x, y)>0\}$. Notice that on $\Gamma, \vec{\eta} \cdot \nabla u<0$ locally to the right (or after) and $\vec{\eta} \cdot \nabla u>0$ locally to the left (or before) $\left(x_{0}, y_{0}\right)$; hence $\left(x_{0}, y_{0}\right) \in \overline{W_{+}} \cap \overline{W_{-}}$. Let $\omega_{1}$ be a component of $W_{+}$whose closure contains $\left(x_{0}, y_{0}\right)$ and let $\gamma_{1}$ be the component of $\partial \omega_{1} \cap \Omega$ whose closure contains $\left(x_{0}, y_{0}\right)$. Let us orient $\gamma_{1}$ so that $\omega_{1}$ lies to the right of $\gamma_{1}$; that is, so that $\left(x_{0}, y_{0}\right)$ is the initial point of $\gamma_{1}$. By Lemma $6, \phi$ is strictly increasing on $\gamma_{1}$, $\overline{\gamma_{1}} \cap \Gamma^{*} \neq \varnothing$, and $\vec{\eta} \cdot \nabla u=0$ on $\gamma_{1}$. Notice that $\gamma_{1} \subset \partial W_{-}$, since 
otherwise we obtain a contradiction of the strong maximum principle (e.g. the third paragraph of the proof of Theorem 1).

Let $\left(x_{1}, y_{1}\right) \in \overline{\gamma_{1}} \cap \Gamma^{*}$ and notice that $\vec{n}\left(x_{1}, y_{1}\right)=\vec{\nu}$. If $\left(x_{1}, y_{1}\right)$ is a $\vec{\nu}$-minimum of $\Gamma^{*}$ and $\overline{\omega_{1}}$ contains a portion of $\Gamma^{*}$ to the right of $\left(x_{1}, y_{1}\right)$, we stop. Otherwise we continue $\gamma_{1}$ beyond $\left(x_{1}, y_{1}\right)$. If $\left(x_{1}, y_{1}\right)$ is not a positive inflection point of $\Gamma^{*}$, then either $\vec{\eta} \cdot \nabla u>0$ locally to the right of $\left(x_{1}, y_{1}\right)$ on $\Gamma^{*}$ or there is a line segment $\sigma \subset \Gamma^{*}$ with $\left(x_{1}, y_{1}\right) \in \sigma$ and $\vec{\eta} \cdot \nabla u>0$ locally to the right of $\sigma$ on $\Gamma^{*}$. If $\left(x_{1}, y_{1}\right)$ is a positive inflection point of $\Gamma^{*}$, then $\vec{\eta} \cdot \nabla u<0$ locally to the left of $\left(x_{1}, y_{1}\right)$ or locally to the left of a line segment $\sigma \subset \Gamma^{*}$ with $\left(x_{1}, y_{1}\right) \in \sigma$. We will describe a rule for obtaining a set $\gamma$ with $\gamma_{1} \subset \gamma$ such that $\gamma$ begins at $\left(x_{0}, y_{0}\right)$ and ends at a $\vec{\nu}$-minimum of $\Gamma^{*}$.

Suppose $\left(x_{1}, y_{1}\right)$ is not a positive inflection point of $\Gamma^{*}$. Let $\sigma$ be the (possibly degenerate) maximal closed line segment such that $\left(x_{1}, y_{1}\right) \in \sigma \subset \Gamma^{*}$ and let $z_{1}, z_{2}$ be the initial and terminal endpoints of $\sigma$ respectively (with $z_{1}=z_{2}$ if $\sigma$ is degenerate). By the corollary to Lemma 1 , we see that $\overline{\omega_{1}} \cap \sigma \subseteq\left\{z_{1}, z_{2}\right\}$ and if $z_{1} \neq z_{2}$, then $\phi\left(z_{1}\right)>\phi\left(z_{2}\right)$. If $\bar{\omega} \cap \sigma=\left\{z_{1}, z_{2}\right\}, z_{1} \neq z_{2}$, and $\xi$ is the component of $W_{-}$whose closure contains $\sigma$, then $\gamma=\partial \xi \cap \Omega$ satisfies $\bar{\gamma} \cap$ $\partial \Omega=\left\{z_{1}, z_{2}\right\}$ and $\vec{\eta} \cdot \nabla u=0$ on $\gamma$; since $\xi$ lies to the left of $\gamma$ as $(x, y) \in \gamma$ moves from $z_{1}$ to $z_{2}, \phi\left(z_{1}\right)<\phi\left(z_{2}\right)$, which is a contradiction. Thus $\overline{\omega_{1}} \cap \sigma=\left\{z_{0}\right\}$, where $z_{0}$ is $z_{1}$ or $z_{2}$, and $\overline{\omega_{1}}$ does not intersect the portion of $\Gamma^{*}$ locally to the right of $z_{0}$. Let $\omega_{\varepsilon}$ be the component of $\omega_{1} \cap B\left(z_{0}, \varepsilon\right)$ which satisfies $\partial \omega_{\varepsilon} \cap \gamma_{1} \cap \Omega \neq \varnothing$, for each $\varepsilon>0$. Let $\gamma_{2}$ be the component of $\partial \omega \cap \Omega$ which is disjoint from $\gamma_{1}$ and satisfies $\gamma_{2} \cap \partial \omega_{\varepsilon} \neq \varnothing$ for each $\varepsilon>0$. Notice that $\gamma_{2}$ is the component of $\partial \omega_{1} \cap \Omega$ "immediately to the right" of $\gamma_{1}$. From Lemma 6 , we see that $\phi>\lambda^{2}$ on $\overline{\gamma_{2}}$ and so $\overline{\gamma_{2}} \cap \Gamma=\varnothing$. Let us orient $\gamma_{2}$ so that $\omega_{1}$ is to the right of $\gamma_{2}$. Then as $(x, y) \in \gamma_{2}$ leaves $z_{0}, \phi(x, y)$ increases (strictly). Notice that there is no open set $\tilde{\omega} \subset \Omega$ with $\partial \tilde{\omega} \subset \overline{\gamma_{2}}$ (i.e. $\gamma_{2}$ cannot cross itself) because of the strict monotonicity of $\phi$. Therefore $\Gamma^{*} \cap \overline{\gamma_{2}} \backslash \sigma \neq \varnothing$.

Suppose $\left(x_{1}, y_{1}\right)$ is a positive inflection point of $\Gamma^{*}$. Let $\sigma$ be the (possibly degenerate) maximal closed line segment satisfying $\left(x_{1}, y_{1}\right)$ $\in \sigma \subset \Gamma^{*}$ and let $z_{1}$ and $z_{2}$ be the initial and terminal endpoints of $\sigma$ respectively. As above, $\overline{\omega_{1}} \cap \sigma=\left\{z_{0}\right\}$, where $z_{0}=\left(x_{1}, y_{1}\right)$ is either $z_{1}$ or $z_{2}$, and $\phi\left(z_{1}\right)>\phi\left(z_{2}\right)$ if $z_{1} \neq z_{2}$. Since $\vec{\eta} \cdot \nabla u<0$ locally to the left of $z_{1}, \overline{\omega_{1}}$ does not intersect the portion of $\Gamma^{*}$ locally to the left of $z_{1}$. 
Suppose $z_{1} \neq z_{2}$ and let $\omega_{0}$ be the component of $W_{-}$whose closure contains $\sigma$. Let $\lambda_{1}$ and $\lambda_{2}$ be the components of $\partial \omega_{0} \cap \Omega$ with $z_{2} \in \overline{\lambda_{1}}$ and $z_{1} \in \overline{\lambda_{2}}$ respectively such that $\partial \omega_{\varepsilon} \cap \partial \omega_{0} \subseteq$ $\lambda_{1} \cup \sigma \cup \lambda_{2}$ for all sufficiently small $\varepsilon>0$, where $\omega_{\varepsilon}$ is the component of $\left\{(x, y) \in \omega_{0}: \operatorname{dist}((x, y), \sigma)<\varepsilon\right\}$ whose closure contains $\sigma$. Suppose also $z_{0}=z_{2}$ and $\gamma_{1} \cap \gamma_{1} \neq \varnothing$. Then $\gamma_{1} \cap B\left(z_{2}, \varepsilon\right) \subseteq \lambda_{1}$ for some $\varepsilon>0$. Let us define $\gamma_{2}=\sigma \cup \lambda_{2}$ and orient $\gamma_{2}$ beginning at $z_{2}$ and going toward (and beyond) $z_{1}$.

Suppose now $z_{0}=z_{1}$ or $z_{0} \neq z_{1}$ and $\gamma_{1} \cap \lambda_{1}=\varnothing$. Let $y^{0} \in \gamma_{1} \cap E$; by Lemma $1, \vec{\eta} \cdot \nabla u<0$ to the right of $\gamma_{1}$ near $y^{0}$ and $\vec{\eta} \cdot \nabla u>\theta$ to left of $\gamma_{1}$ near $y^{0}$. Let $\left(y^{n}\right)$ be a collection of points after $y^{0}$ on $\gamma_{1}$, given in increasing order on $\gamma_{1}$, at which the condition $\vec{\eta} \cdot \nabla u>0$ to the left of $\gamma_{1}$ near $y^{n}$ is not satisfied. By Lemma 2, for each $n$, there exists $z^{n} \in \gamma_{1} \cap E$ between $y^{n-1}$ and $y^{n}$ and so $\vec{\eta} \cdot \nabla u>0$ to the left of $\gamma_{1}$ near $z^{n}$. Then there is a component $w^{n}$ of $W_{+}$with $y^{n} \in \partial w^{n}$ such that $w^{n}$ lies to the left of $\gamma_{1}$ near $y^{n}$ for each $n$. Let $\lambda^{n}$ be a component of $\partial w^{n} \backslash\left(\gamma_{1} \cup \partial \Omega\right)$, oriented to begin at $y^{n}$, such that $w^{n}$ lies to the right of $\lambda^{n}$ and so $\phi$ is strictly increasing on $\lambda^{n}$. Now $\overline{\lambda^{n}} \cap \Gamma=\varnothing$ and so $\overline{\lambda^{n}}$ must interest $\Gamma^{*}$. Suppose $\overline{\lambda^{m}}$ and $\overline{\lambda^{n}}$ either intersect one another or intersect the same component of $\Gamma_{\vec{\eta}}^{*}=\left\{(x, y) \in \Gamma^{*}: \vec{\eta} \cdot \nabla(x, y)=0\right\}$. Then the component $U_{m n}$ of $\Omega \backslash\left(\lambda^{m} \cup \lambda^{n} \cup \gamma_{1}\right)$ whose closure contains $\lambda^{m} \cup \lambda^{n}$ must enclose a component of $\Gamma^{*}$ because otherwise the strong maximum principle yields a contradiction. Since $\Gamma^{*}$ has only a finite number of components, only a finite number of pairs $(m, n)$ can satisfy the last supposition. Since $\Gamma_{\vec{\nu}}^{*}$ has a finite number of components, there can only be a finite number of points $\left(y^{n}\right)$ as supposed and hence $\vec{\nu} \cdot \nabla u>0$ locally to the left of $\gamma_{1}$ in a neighborhood of $\gamma_{1} \cap B\left(z_{0}, \varepsilon_{0}\right)$ for some $\varepsilon_{0}>0$. Let $\omega_{2}$ be the component of $W_{-}$whose closure contains $\overline{\gamma_{1}} \cap B\left(z_{0}, \varepsilon_{0}\right)$. Notice that if $z_{0}=z_{2} \neq z_{1}$, then $\omega_{2} \cap \omega_{0}=\varnothing$ (at least in a neighborhood of $\sigma$ ). Now let $\gamma_{2}$ be the component of $\partial \omega_{2} \cap \Omega$ whose closure contains $z_{0}$ and which lies immediately to the left of $\gamma_{1}$ on $\partial \omega_{2}$. Let us orient $\gamma_{2}$ to begin at $z_{0}$.

Let us review our procedure. If $\left(x_{1}, y_{1}\right)$ is a $\vec{\nu}$-minimum of $\Gamma^{*}$ and the component of $\partial \omega_{1} \cap \Omega$ immediately to the right of $\gamma_{1}$ is a portion of $\Gamma^{*}$, we terminate our procedure. Otherwise, we see that there exists a set $\gamma_{2}$ beginning at $\left(x_{1}, y_{1}\right)$ on which $\vec{\eta} \cdot \nabla u=0$ and $\phi$ is strictly increasing. In particular, $\gamma_{2}$ is the component of $\partial \omega_{1} \cap \Omega$ immediately to the right of $\gamma_{1}$ if $\left(x_{1}, y_{1}\right)$ is not a positive inflection point of $\Gamma^{*}$ and $\gamma_{2} \backslash \sigma$ is the component of $\partial \omega_{2} \cap \Omega$ im- 
mediately to the left of $\gamma_{1}$ if $\left(x_{1}, y_{1}\right)$ is a positive inflection point of $\Gamma^{*}$, where $\omega_{2}$ is the component of $W_{-}$immediately to the left of $\gamma_{1}$ near $\left(x_{1}, y_{1}\right)$. We may continue this process to obtain points $\left(x_{1}, y_{1}\right),\left(x_{2}, y_{2}\right), \ldots,\left(x_{n}, y_{n}\right)$ and a set $\gamma=\gamma_{1} \cup \gamma_{2} \cup \cdots \cup \gamma_{n}$ such that $\phi$ is strictly increasing on $\gamma$ and $\vec{\eta} \cdot \nabla u=0$ on $\gamma$. Due to the monotonicity of $\phi$ and the maximum principle, $\gamma$ cannot cross itself and $\left(x_{i}, y_{i}\right)$ and $\left(x_{j}, y_{j}\right)$ can be elements of the same component $\sigma$ of $\Gamma_{\vec{\nu}}^{*}$ only if $\sigma$ is a nondegenerate line segment, $\left(x_{i}, y_{i}\right)$ is the terminal endpoint of $\sigma,\left(x_{j}, y_{j}\right)$ is the initial endpoint of $\sigma$, and $\gamma_{i+1} \cup \cdots \cup \gamma_{j}$ surrounds a component of $\partial \Omega$. Since $\Gamma_{\vec{\nu}}^{*}$ and $\partial \Omega$ each have a finite number, say $M$ and $N$, of components, this process must terminate after a finite number $(\leq M+N)$ of steps. On the other hand, the process cannot terminate except at a $\vec{\nu}$-minimum of $\Gamma^{*}$, since we can continue $\gamma$ past $\left(x_{n}, y_{n}\right)$ if $\left(x_{n}, y_{n}\right)$ is not a $\vec{\nu}$ minimum of $\Gamma^{*}$. Hence $\gamma$ terminates at a $\vec{\nu}$-minimum of $\Gamma^{*}$. From the rules used for continuation, we see that two curves $\gamma$ and $\tilde{\gamma}$ cannot terminate at the same point unless $\gamma_{i}=\tilde{\gamma}_{j}$ for some $i$ and $j$. However, this cannot occur unless $\gamma$ and $\tilde{\gamma}$ begin at the same point. The fact that the total positive curvature of $\Gamma$ is bounded by the total positive curvature of $\Gamma^{*}$ follows as in the proof of Theorem 2 of [4].

The proof of Theorem 4 follows from the proof of Theorem 3 above or from the proof of Theorem 5 of [4].

REMARK. In [11], Friedman and Vogel examined two-dimensional ideal fluid flows with a cavity behind an obstacle in an infinite channel with an oscillatory wall. One aspect of their work involved determining some geometric aspects of the free boundary and used curves of constant gradient direction. Using the ideas of this paper, especially Lemma 1, geometric properties of two-dimensional, compressible, irrotational, inviscid, subsonic cavitation flows (e.g. [18, p. 109]) past an obstacle in an infinite, oscillatory channel could be determined. When $\Omega$ is assumed bounded, the results of this section apply directly to our free boundary problems when, for example, $(2 \mathrm{a})$ or $\left(2 \mathrm{a}^{\prime}\right)$ is the minimal surface equation, the $p$-Laplace equation, or Pucci's equation ([12]). When $\Omega$ is not assumed bounded, we can still obtain qualitative information about the free boundary in the same manner as for compressible flows. 


\section{REFERENCES}

[1] A. Acker, On the geometric form of free boundaries satisfying a Bernoulli condition, Math. Methods Appl. Sci., 6 (1984), 449-456.

[2] _ On the geometric form of free boundaries satisfying a Bernoulli condition. II, Math. Methods Appl. Sci., 8 (1986), 387-404.

[3] __, On the geometric form of solutions of a free boundary problem involving galvanization, Math. Methods Appl. Sci., 9 (1987), 99-104.

[4] - On the geometric form of Bernoulli configurations, Math. Methods Appl. Sci., 10 (1988), 1-14.

[5] __, On the geometric form of axial-symmetric free boundaries satisfying a Bernoulli condition, manuscript.

[6] A. Acker and K. Lancaster, The geometry of curves of constant contact angle for doubly-connected minimal surfaces, Comm. Partial Differential Equations, 14 (1989), 375-390.

[7] __ Existence and geometry of a free boundary problem for the heat equation, Pacific J. Math., 148 (1991), 207-224.

[8] A. Beurling, The Collected Works of Arne Beurling, Vol. 1, Boston: Birkhäuser, 1989.

[9] R. Courant and D. Hilbert, Methods of Mathematical Physics, Vol. 1, New York: Springer, 1970.

[10] A. Friedman and R. Jensen, Convexity of the free boundary in the Stefan problem and in the dam problem, Arch. Rational Mech. Anal., 67 (1978), 1-24.

[11] A. Friedman and T. Vogel, Cavitational flow in a channel with oscillatory wall, Nonlinear Anal. TMA, 7 (1983), 1175-1192.

[12] D. Gilbarg and N. Trudinger, Elliptic Partial Differential Equations of Second Order, second edition, Berlin: Springer, 1983.

[13] K. Lancaster, Partial continuity at reentrant corners for Perron solutions of Dirichlet problems with certain boundary data, manuscript.

[14] K. Nickel, Einige Eigenschaften von Lösungen der Prandtlschen GrenzschichtDifferentialgleichungen, Arch. Rational Mech. Anal., 2 (1958), 1-31.

[15] _ Gestaltaussagen Uber Losungen parabolischer Differentialgleichungen, J. Reine Angew. Math., 211 (1962), 78-94.

[16] J. C. C. Nitsche, Lectures on Minimal Surfaces, Vol. 1, Cambridge: Cambridge Univ. Press, 1989.

[17] L. Payne, On two conjectures in the fixed membrane eigenvalue problem, J. Appl. Math. Phys. (ZAMP), 24 (1973), 721-729.

[18] M. Schiffer, Analytic Theory of Subsonic and Supersonic Flows, 1-161: Encylopedia of Physics 9, Berlin: Springer, 1960.

[19] J. Serrin, A priori estimates for solutions of the minimal surface equation, Arch. Rational Mech. Anal., 14 (1963), 376-383.

[20] D. Tepper, Free boundary problem, SIAM J. Math. Anal., 5 (1974), 841-846.

[21] - Free boundary problem - the starlike case, SIAM J. Math. Anal., 6 (1975), 503-505.

[22] T. Vogel, $A$ free boundary problem arising from a galvanizing process, SIAM J. Math. Mech. Anal., 16 (1985), 970-979.

Received March 13, 1991. 


\section{PACIFIC JOURNAL OF MATHEMATICS EDITORS}

\author{
V. S. VARADARAJAN \\ (Managing Editor) \\ University of California \\ Los Angeles, CA 90024-1555 \\ Herbert Clemens \\ University of Utah \\ Salt Lake City, UT 84112 \\ F. Michael Christ \\ University of California \\ Los Angeles, CA 90024-1555 \\ THOMAS ENRIGHT \\ University of California, San Diego \\ La Jolla, CA 92093
}

\author{
Nicholas ERcolani \\ University of Arizona \\ Tucson, AZ 85721 \\ R. FINN \\ Stanford University \\ Stanford, CA 94305 \\ VAughan F. R. JONES \\ University of California \\ Berkeley, CA 94720 \\ C. C. Moore \\ University of California \\ Berkeley, CA 94720 \\ MARTIN ScharlemanN \\ University of California \\ Santa Barbara, CA 93106 \\ Harold Stark \\ University of California, San Diego \\ La Jolla, CA 92093
}

STEVEN KeRCKHOFF

Stanford University

Stanford, CA 94305

\begin{tabular}{|c|c|c|c|c|}
\hline \multicolumn{5}{|c|}{ ASSOCIATE EDITORS } \\
\hline R. Arens & $\begin{array}{ll}\text { E. F. BECKENBACH } & \text { B. H } \\
(1906-1982) & \end{array}$ & NeUmanN & $\begin{array}{c}\text { F. WoLF } \\
(1904-1989)\end{array}$ & K. Yoshida \\
\hline \multicolumn{5}{|c|}{ SUPPORTING INSTITUTIONS } \\
\hline \multicolumn{2}{|c|}{ UNIVERSITY OF ARIZONA } & \multicolumn{3}{|c|}{ UNIVERSITY OF OREGON } \\
\hline \multicolumn{2}{|c|}{ UNIVERSITY OF BRITISH COLUMBIA } & \multicolumn{3}{|c|}{ UNIVERSITY OF SOUTHERN CALIFORNIA } \\
\hline \multicolumn{2}{|c|}{ CALIFORNIA INSTITUTE OF TECHNOLOGY } & \multicolumn{3}{|c|}{ STANFORD UNIVERSITY } \\
\hline \multicolumn{2}{|c|}{ UNIVERSITY OF CALIFORNIA } & \multicolumn{3}{|c|}{ UNIVERSITY OF HAWAII } \\
\hline \multicolumn{2}{|c|}{ MONTANA STATE UNIVERSITY } & \multicolumn{3}{|c|}{ UNIVERSITY OF TOKYO } \\
\hline \multicolumn{2}{|c|}{ UNIVERSITY OF NEVADA, RENO } & \multicolumn{3}{|c|}{ UNIVERSITY OF UTAH } \\
\hline & \multicolumn{3}{|c|}{ WASHINGTON STATE UNIVERSITY } \\
\hline \multicolumn{2}{|c|}{ OREGON STATE UNIVERSITY } & \multicolumn{3}{|c|}{ UNIVERSITY OF WASHINGTON } \\
\hline
\end{tabular}

The Supporting Institutions listed above contribute to the cost of publication of this Journal, but they are not owners or publishers and have no responsibility for its content or policies.

Mathematical papers intended for publication in the Pacific Journal of Mathematics should be in typed
form or offset-reproduced (not dittoed), double spaced with large margins. Please do not use built up fractions
in the text of the manuscript. However, you may use them in the displayed equations. Underline Greek letters
in red, German in green, and script in blue. The first paragraph must be capable of being used separately as
a synopsis of the entire paper. In particular it should contain no bibliographic references. Please propose a
heading for the odd numbered pages of less than 35 characters. Manuscripts, in triplicate, may be sent to any
one of the editors. Please classify according to the 1991 Mathematics Subject Classification scheme which
can be found in the December index volumes of Mathematical Reviews. Supply name and address of author
to whom proofs should be sent. All other communications should be addressed to the managing editor, or
Elaine Barth, University of California, Los Angeles, California $90024-1555-05$.
There are page-charges associated with articles appearing in the Pacific Journal of Mathematics. These
charges are expected to be paid by the author's University, Government Agency or Company. If the author or
authors do not have access to such Institutional support these charges are waived. Single authors will receive
50 free reprints; joint authors will receive a total of 100 free reprints. Additional copies may be obtained at
cost in multiples of 50 .

The Pacific Journal of Mathematics (ISSN 0030-8730) is published monthly except for July and August. Regular subscription rate: $\$ 190.00$ a year (10 issues). Special rate: $\$ 95.00$ a year to individual members of supporting institutions.

Subscriptions, orders for numbers issued in the last three calendar years, and changes of address should be sent to Pacific Journal of Mathematics, P.O. Box 969, Carmel Valley, CA 93924, U.S.A. Old back numbers obtainable from Kraus Periodicals Co., Route 100, Millwood, NY 10546.

The Pacific Journal of Mathematics at P.O. Box 969, Carmel Valley, CA 93924 (ISSN 0030-8730) is published monthly except for July and August. Second-class postage paid at Carmel Valley, California 93924, and additional mailing offices. Postmaster: send address changes to Pacific Journal of Mathematics, P.O. Box 969, Carmel Valley, CA 93924.

PUBLISHED BY PACIFIC JOURNAL OF MATHEMATICS, A NON-PROFIT CORPORATION Copyright (C) 1992 by Pacific Journal of Mathematics 


\section{Pacific Journal of Mathematics}

\section{Vol. 154, No. $2 \quad$ June, 1992}

Manuel (Rodriguez) de León, J. A. Oubiña, P. R. Rodrigues and

Modesto R. Salgado, Almost $s$-tangent manifolds of higher order . . . . 201

Martin Engman, New spectral characterization theorems for $S^{2} \ldots \ldots \ldots 215$

Yuval Zvi Flicker, The adjoint representation $L$-function for GL $(n) \ldots \ldots 231$

Enrique Alberto Gonzalez-Velasco and Lee Kenneth Jones, On the range

of an unbounded partly atomic vector-valued measure . ............ 245

Takayuki Hibi, Face number inequalities for matroid complexes and

Cohen-Macaulay types of Stanley-Reisner rings of distributive

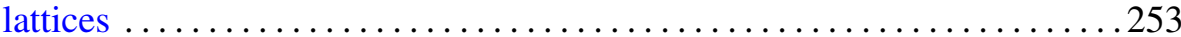

Hervé Jacquet and Stephen James Rallis, Kloosterman integrals for skew

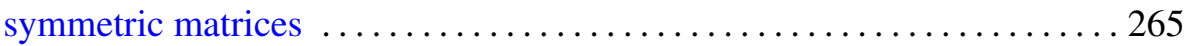

Shulim Kaliman, Two remarks on polynomials in two variables .........285

Kirk Lancaster, Qualitative behavior of solutions of elliptic free boundary problems ..........................................297

Feng Luo, Actions of finite groups on knot complements . ........... 317

James Joseph Madden and Charles Madison Stanton, One-dimensional

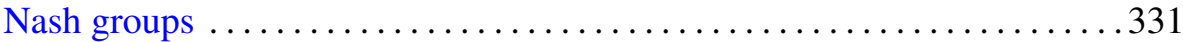

Christopher K. McCord, Estimating Nielsen numbers on

infrasolvmanifolds ......................................... 345

Gordan Savin, On the tensor product of theta representations of $\mathrm{GL}_{3}$ 369

Gerold Wagner, On means of distances on the surface of a sphere. II.

(Upper bounds) 